\title{
La juventud en el trabajo: inequidad y discriminación
}

\author{
Ana María de la Jara* \\ Fabiola Serna Santamaría**
}

\section{RESUMEN}

A partir de los resultados de tres investigaciones, una cuantitativa y dos cualitativas, producidas en el marco de la campaña "más respeto a los derechos laborales de los jóvenes", el presente artículo tiene por objetivo problematizar el discurso público sobre juventud y trabajo. Se evidencia la invisibilización de aspectos como la inequidad y la discriminación que sufren los jóvenes en el trabajo.

Palabras Clave: Juventud / Trabajo decente / Abuso de poder / Derechos laborales.

\section{A juventude no trabalho: inequidad e discriminação}

\section{RESUMO}

A partir dos resultados de três investigações, uma cuantitativa e duas cualitativas, produzidas no marco a campanha "Mais respeito aos direitos trabalhistas dos jovens", o presente artigo tem por objectivo problematizar o discurso público sobre juventude e trabalho. Se evidência a invisibilización de aspectos como a inequidad e a discriminação que sofrem os jovens no trabalho.

Palabras chave: Juventude / Trabalho decente / Abuso de poder / Direitos trabalhistas.

\section{Youth at work: inequality and discrimination}

\section{ABSTRACT}

From the results of three investigations, one quantitative and two qualitative, all conducted under the campaign "More respect for the rights of young women and men at work", this article aims to problematize the public discourse on youth and work. It demonstrates the invisibility of issues such as inequality and discrimination faced by young people at work.

Key Words: Youth / decent work / Abuse of power / Labor rights.

* Chilena, trabajadora social. Directora de Redes y Movilización ONG Genera. Correo electrónico: am.delajara@generaenlinea.cl

** Colombiana, trabajadora social, magister en Ética y Desarrollo Humano. Docente Escuela de Trabajo Social, Universidad Católica Silva Henríquez. Correo electrónico: Fabiola.serna@gmail.com. 


\section{Introducción}

Las reflexiones presentadas en este artículo se enmarcan en los resultados de investigación producidos en la campaña "Más respeto a los derechos laborales de los jóvenes", iniciativa auspiciada por la Unión Europea y desarrollada durante el periodo 2009 - 2010, cuyo propósito es generar información de carácter cualitativo y cuantitativo con el fin de comprender los problemas que enfrenta la juventud en el mundo laboral y potenciar acciones de incidencia social y política con el fin de instalar en la agenda pública el tema del acceso y permanencia de la juventud en el trabajo, como un problema social relevante. Las organizaciones que lideraron este proyecto corresponden a Genera ${ }^{1}$, Escuela de Trabajo Social de la Universidad Silva Henríquez, Ong Cordillera² Sodem ${ }^{3}$ y Achnu ${ }^{4}$.

En el contexto de la iniciativa mencionada, se han realizado dos investigaciones cualitativas tituladas: "Vulneración de los derechos laborales de los jóvenes y políticas públicas de empleo: Panorama y evaluación” (Najarí y Piña, 2009) y “Experiencias y derechos laborales de jóvenes entre 20 y 24 años de la Región Metropolitana" (Peña y Gajardo, 2009). También se realizó un estudio de opinión basado en la aplicación de una encuesta con 500 casos en comunas urbanas y rurales de la Región Metropolitana. El modelo de campaña es básicamente una estrategia de educación ciudadana, en la cual la producción de datos juega un papel relevante para la instalación de los temas en la agenda social y política.

Otro aspecto relevante para esta introducción, se refiere a la importancia de las y los jóvenes como sujetos educativos y como participantes en procesos sociales impulsados por profesionales del Trabajo Social y otros. En este sentido, el artículo sugiere un ejercicio de revisión de los lugares desde los cuales significamos a la juventud.

\section{Nociones sobre Juventud}

Existe en la literatura, una pluralidad de nociones sobre la categoría juventud, la cual ha sido abordada como tramo etario,

\footnotetext{
Paramás detallesver:Genera: Ideasyaccionescolectivas. http://www.generaenlinea.cl/blog/ Para más detalles ver: Ong cordillera: http://www.ongcordillera.cl/

Para más detalles ver: Sodem: Solidaridad y desarrollo: http://www.sodem.cl/

Para más detalles ver: Asociación Chilena Pro Naciones Unidas. Protegiendo los derechos de niñ@s y jóvenes. http://www.achnu.cl/
} 
como construcción cultural, como sujeto social e histórico generador de una cultura propia ó como proceso de transición a la vida adulta. Sin embargo, la idea de la juventud como periodo de transición, aparece con mucha fuerza en las representaciones sociales, como un estado incompleto, que se define desde sus límites con infancia o la adultez (Serrano, 1995, en Peña y Gajardo, 2009).

Las condiciones de "estadio" que se unen a las transformaciones biológicas y psicológicas, provocan necesariamente una reestructuración de la personalidad, orientada a la adaptación al medio social. En efecto, estos procesos de desarrollo biológico y psicológico suponen un estado de maduración personal que explica la construcción de una mirada hacia los jóvenes como pre-adultos y favorece la tendencia a la asociación de la juventud con la carencia o el déficit, con una crisis de identidad y conflictos internos, lo que entrega una imagen de los jóvenes como un grupo cuya meta normativa y deber moral de adaptación a la sociedad es llegar a la adultez.

Sin embargo, la constante petición de atributos que tienden a ese estadio adulto, demanda a los jóvenes una serie de responsabilidades y ambiciones personales, que chocan con la dependencia y estancamiento social a los cuales generalmente están sometidos, quedando posicionados con frecuencia como víctimas de las condiciones sociales (Serrano, 1995, en Peña y Gajardo, 2009).

\section{Jóvenes y Acceso al Trabajo}

Son conocidos los indicadores acerca de las dificultades que las y los jóvenes experimentan para insertarse y mantenerse en el mercado del trabajo en todos los países de América Latina, donde las y los jóvenes representan el 46\% del total de desempleados. (CEPAL (2004). Por otra parte, de acuerdo con Weller (2006), si nos situamos en el contexto de las crisis, los datos reflejan que es precisamente este grupo etario el que se constituye como la variable más rápida de ajuste en las empresas, siendo parte de un mercado laboral con empleos de corto plazo y por ende más inestables.

Aunque los indicadores podrían leerse como evidencia de la natural valoración de la experiencia en el mercado laboral, la hipótesis de este artículo es que en el mundo del trabajo se reproduce la 
inequidad social y se revelan conductas discriminatorias hacia las y los jóvenes que pertenecen a los niveles socioeconómicos medios y bajos.

Al respecto, la quinta encuesta nacional de juventud muestra que el $48 \%$ de las y los jóvenes, han estudiado la enseñanza básica en colegios municipales, dos tercios de ellos se encuentran "solo trabajando", "sólo buscando trabajo" o "no estudiando ni trabajando". Quienes combinan estudio y trabajo o se dedican solo al estudio, provienen en su mayoría de establecimientos particulares subvencionados o particulares pagados; en las y los jóvenes del nivel socioeconómico alto $(A B C 1)$, se encuentran quienes no han trabajado nunca ni están buscando trabajo, junto con quienes sí trabajan y la condición de desempleo es menor que en los demás niveles socioeconómicos (INJUV 2007:43). Esta situación refleja que la sabida inequidad del sistema educativo, tiene un correlato en el acceso a oportunidades de trabajo decente, comprendido como un trabajo productivo en condiciones de libertad, igualdad, seguridad y dignidad humana. (OIT, 1996)

De otro lado, la encuesta CASEN (2006), muestra que las áreas de empleo en donde hay mayor cantidad de jóvenes con ingresos bajos, es en la de trabajadores no calificados $(98,1 \%)$, y en vendedores de comercio (94,3\%). Mientras que son los jóvenes entre 20 y 24 años los que tienen menor cantidad de contratos indefinidos $(55,4 \%)$. El tipo de relación contractual puede ser observada por la pertenencia de las y los jóvenes a un quintil de ingresos; la población joven perteneciente al I quintil posee menor proporción de contratos a plazo indefinido (45\%). En relación con el resto de los quintiles juveniles, son el grupo que posee mayormente contratos de tipo por obra, faena o servicio (30\%), los cuales son los más precarios y los que menor estabilidad laboral le entregan a un empleado.

Por su parte, la población joven perteneciente al V quintil, es la que más posee contrato a plazo indefinido (80\%), y a su vez la que menos lo posee por obra, faena o servicio $(5,7 \%$ ) (Najari y Piña, 2008:61). Entretanto, un 43,3\% de los jóvenes con ingresos altos realizan horas extras por decisión propia, mientras que un 98,8\% de los jóvenes con ingresos bajos las realizan porque un jefe lo determina (Encuesta Trabajo y Equidad 2008).

Presentadosalgunosindicadoressobreaccesoaltrabajo, es posible afirmar que la variable inequidad se encuentra invisibilizada en el discurso público sobre juventud, por varias razones: la primera 
de ellas es que a pesar de que el desempleo juvenil aparece con frecuencia en la agenda pública, las soluciones frecuentes como subsidios a la contratación o planes de capacitación y empleo, son medidas de corto plazo que omiten la revisión de la oferta educativa y laboral que la sociedad, en su conjunto, entrega a esos dos tercios de las y los jóvenes que estudiaron en el sistema municipalizado y se encuentran trabajando en contextos precarios, buscando trabajo o desocupados.

\section{Discriminación: Un tema Cultural}

Otra de las razones de la invisibilización de la inequidad y la discriminación en el discurso público sobre jóvenes y trabajo tiene que ver con las percepciones de los empleadores respecto de este segmento de jóvenes, cuyo capital cultural es débil, su experiencia laboral escasa, su conocimiento de las normas básicas de la cultura laboral y su apariencia no son muy adecuadas. (Injuv, 2007)

Al respecto, el estudio jóvenes y trabajo (Genera, 2009) muestra la existencia de opiniones negativas acerca de las y los jóvenes en relación con afirmaciones como: "los trabajadores adultos se sienten amenazados por trabajadores jóvenes", donde el 71\% de la población está de acuerdo. "Los jóvenes son descuidados o poco prolijos en el trabajo", donde existe un 59\% acuerdo. "Los jóvenes tienen menos responsabilidad que los adultos en el trabajo" (58\% acuerdo) y "los jóvenes son flojos en el trabajo" (57\% acuerdo).

Es interesante observar en el discurso de las y los jóvenes entrevistados y en el estudio de Peña y Gajardo (2009), la existencia de una explicación alternativa frente a la opinión generalizada, sobre todo de los adultos, de que los jóvenes son flojos en el trabajo. Estas conductas que se plantean en algunos casos como asumidas, responderían no solo a un choque con el mundo de los adultos, sino también a ofertas laborales caracterizadas por la precariedad, tal como lo muestran las siguientes citas:

“Los jóvenes se meten a un mercado laboral, mal pagado y todo, no sé po’, una cuestión mucho más estática en términos de proyecciones laborales. Pegas mecánicas y a la vez la gente retroalimenta una pega penca, uno no se entrega bien, es negligente, saca la vuelta porque la pega es fome..." (H6, urbano). 
"Para un joven es más fácil acceder a puestos más precarios, que son los que más abundan porque se les puede pagar menos. Un adulto no va a aceptar" (H6, urbano).

La brecha socio-económica se evidencia en otros aspectos como el tiempo necesario para encontrar el primer empleo. Dentro de los jóvenes pertenecientes al nivel socioeconómico ABC1, un 38,8\% declaró "no haberse demorado nada" en encontrar su primer trabajo, y un 0\% declaró haberse demorado más de un año, es decir, virtualmente ningún caso. En cambio, en el estrato E, un 21,2\% declaró haberse demorado nada, y un 12,3\% más de un año (Injuv, 2007).

Las opiniones registradas por la encuesta Jóvenes y Trabajo, muestran la opinión de la población respecto de que los principales aspectos en los que se fijan los empleadores para contratar jóvenes corresponden a: el lugar donde estudió (27\%); sus recomendaciones (21\%); que sea recomendado por un conocido (18\%) y su apariencia física (13\%).

Al respecto, las opiniones de las y los jóvenes de nivel socioeconómico medio y bajo, confirman desde su experiencia la existencia de barreras frecuentes de entrada al mundo del trabajo, en relación con la escasez de oportunidades:

"A mí me bajoniaba", cuando terminé de estudiar... después de todo lo que me había costado... no poder encontrar trabajo en lo que yo quería, yo sentía que mi esfuerzo no había servido para nada..." (H2, rural).

"Mi mayor problema para encontrar trabajo siempre era que te pedian experiencia y lo peor es que cuando uno es joven, no tení de dónde sacar experiencia po'... si apenas hay trabajo..." (H2, rural).

"Yo me acuerdo bien, que una vez para un verano solo quería trabajar por uno o dos meses y guardar la plata para el año, para los estudios... entonces me dieron un buen dato en la OMIL... fui y me hicieron unas pruebas para ver si quedaba o no... me dijeron altiro que no, ni siquiera sé por qué... quizás me encontraron loco..." (H4, rural).

"La falta de experiencia siempre te pesa... no solo desde la perspectiva de mirada de los jefes respecto a tu trabajo... sino que también desde las trabas que uno mismo se genera por no tener experiencia... lo digo porque uno mismo se asusta y empieza a mermar su capacidad de producir

5 Chilenismo que significa pena o frustración

6 Chilenismo que significa inmediatamente. 
mejores productos en el trabajo, pensando que la falta de experiencia condiciona fuertemente lo que puedes entregar" (H4, urbano).

Además de las barreras de entrada al mercado del trabajo, donde la ausencia de experiencia da origen a un círculo vicioso que refleja la existencia de una cultura laboral que no "enseña", o dicho de otro modo, de un mundo del trabajo poco interesado en la juventud; la interacción con los trabajadores adultos, representa con frecuencia otro signo de discriminación hacia este grupo etario, lo cual puede estar relacionado con condiciones laborales inestables, pero también con las ventajas competitivas de los jóvenes que hoy tienen un mayor nivel educativo que los adultos, y con la vigencia del paradigma adulto - céntrico que se reproduce en el mundo del trabajo:

“Hay un punto importante de que si tú eres nuevo en el trabajo y hay gente mayor o más experiencia trata de apoyarte o ponerte más peso. Por ejemplo tú estái en el trabajo y dicen ya, este compadre es nuevo, enséñale, que empiece a trabajar de a poco. Pero hay mucha gente que trata de ponerte más carga de trabajo y como tú eres pollito chico no podí decir no po', esto es así, este es mi trabajo... y esa gente son los mismos trabajadores, empleadores y eso pasa en todo tipo de empresas". (H2, urbano).

"En la empresa actual se ve que las personas que tienen un cierto rango en la empresa y no tienen mucho estudio, y a veces uno en el estudio aprende otras cosas que te lo enseña la teoría y ve entonces que hay una diferencia, entonces en ese aspecto puede haber cierta envidia, recelo en los estudios" (H2, urbano).

“En el trabajo, hay grupos así, infranqueables, cuesta entrar, y cuesta también hacerles entender que uno no tiene intención alguna de quitarles la pega... Por el contrario, solo quiere aportar a la empresa con lo que uno sabe, tal cual ellos lo hicieron cuando ellos entraron..." (H4, urbano).

\section{Mujeres Jóvenes}

No es casual que el acceso de las mujeres jóvenes al trabajo, plantee mayores dificultades que en el caso de los hombres jóvenes, lo cual se refleja en mayores tasas de desempleo y menores salarios. Esto indica que las mujeres, a lo largo de su vida laboral, reciben un menor premio a la experiencia que los hombres, bien sea porque acumulan menos experiencia debido 
a trayectorias laborales interrumpidas, o a discriminaciones salariales (OIT 2006).

El menor acceso relativo a las oportunidades del mercado laboral se agudiza en los segmentos más pobres de la población femenina, de manera tal que las mujeres están sobre-representadas en las categorías de menor valoración social y remuneración, y subrepresentadas entre los empleadores y cargos ejecutivos. Esto es: un $1,9 \%$ de ellas es empleadora, versus un 3,7\% de los hombres; a su vez, un 12,7\% de ellas se dedica al servicio doméstico, frente a solo un $0,2 \%$ de los hombres. Las mujeres, pese a tener en promedio más años de escolaridad que los hombres (11,8 frente a 11,7 ), perciben en promedio el 79\% del sueldo de éstos. Aquella situación se agudiza en los sectores económicamente más vulnerables de la población (Injuv, en Piña y Najarí, 2009).

Respecto del acceso a la educación, dentro de las razones dadas por las y los jóvenes para no estar estudiando, el factor género marca una diferencia importante. Mientras el 24,4\% de los varones señalan como razón para estar fuera del sistema educacional la decisión de trabajar, solo el 8,7\% de las mujeres señala aquello como la causa de su abandono educacional, la cual es reemplazada por "la necesidad de cuidar a su hijo(a)", motivo que alcanza a un $20,3 \%$ en el caso de las mujeres y solo un 1,3\% en el caso de los varones" (Injuv, 2007:52).

En el estudio Jóvenes y Trabajo (Genera, 2009), frente a la pregunta sobre si las mujeres jóvenes embarazadas deben dejar el trabajo para cuidar sus hijos, aproximadamente un tercio de la población cree que es siempre necesario, otro tercio que solo a veces y el tercio restante que no es necesario. En esta respuesta, a medida que avanza la edad, mayor es la proporción de personas que consideran que es necesario que las mujeres jóvenes dejen de trabajar, pasando de $23 \%$ en los jóvenes a prácticamente el doble (44\%) en los adultos mayores.

Este dato aparece con mucha relevancia para el análisis de la inserción laboral de las mujeres jóvenes dado que la polaridad en las respuestas, evidencia la inexistencia de consenso social sobre la definición de roles familiares o más bien la persistencia de la responsabilización exclusiva de las mujeres en la crianza de los hijos y el trabajo doméstico. Este indicador es complementado por las opiniones de las y los encuestados frente a los principales motivos para no contratar a un hombre joven o a una mujer joven. 
En el caso de los hombres jóvenes, los principales motivos para no ser contratados son: no tener experiencia laboral (49\%), ser irresponsables (11\%), ser poco comprometidos con lo que hacen $(8 \%)$, ser desordenados (13\%), no tener disciplina (5\%), ser flojos (5\%) y por que le quitan el trabajo a los adultos (3\%), mientras que en el caso de las mujeres, las principales razones para no ser contratadas son: la poca experiencia laboral (32\%), porque están en edad fértil (26\%), porque son chismosas o copuchentas (8\%), porque son irresponsables $(6 \%)$, son poco comprometidas con lo que hacen (5\%). Queda en evidencia que las construcciones sociales en torno a las relaciones de género castigan de manera particular a las mujeres en el ámbito del trabajo.

Otro aspecto relevante en el análisis de la categoría género dice relación con el acoso sexual. En la pregunta acerca de la naturalización de prácticas laborales, un 46\% de la población piensa que las mujeres son víctimas de acoso sexual, aspecto que evidencia la naturalización de prácticas culturales que violentan sexualmente a las mujeres y se reproducen en el plano laboral. Las siguientes citas ilustran la existencia de conductas en el trabajo que afectan particularmente a las mujeres jóvenes:

“En mi primera pega viví el machismo, viví una situación que es un poco incómoda para las mujeres como es el acoso..." (M2, urbana).

"Yo creo que siempre te miran con desconfianza cuando eres mujer, ya más grande y todo eso... porque les da miedo que quedí embarazá y todo eso... por eso te preguntan altiro si eres casada o no, o por los hijos... en cambio yo creo, a un hombre si le preguntan eso, no les importa y si es igual, igual a ti en condiciones y todo igual, lo van a elegir a él no más..." (M3, urbana).

"Intenté buscar trabajo y no me dieron por el hecho de estar embarazada, porque para ellos se les hace muy complicado pagar el prenatal y el postnatal, porque además el estar en postnatal significa horarios, se sale a otros horarios, a ellos no les conviene contratar a una persona embarazada y aparte que acá en Curacaví ya no hay, si no es para las viñas..." (M1, rural).

“Dejé de trabajar porque me casé y tuve que criar a mis hijos... pero cuando trabajé lo hice simplemente para ganar más dinero y ayudar en mi casa..." (M3, rural). 
"El trabajo acá siempre es escaso, nunca hay muchas oportunidades para trabajar, sobre todo si eres mujer, ahí se vuelve mucho más difícil poder encontrar algo, o sea, algo bueno, decente, me entendí, porque tampoco una puede estar trabajando toda su vida en trabajos de temporera." (M3, rural).

\section{Jóvenes Rurales}

La juventud rural merece una mención especial respecto de su vinculación con el mundo del trabajo. Según la CEPAL (2005), en términos geográficos, la falta de oportunidades de educación y de empleo remunerado en las zonas rurales se traduce en una inserción laboral demasiado temprana sobre todo entre los hombres, con obstáculos a la inserción sobre todo entre las mujeres.

En promedio, las y los jóvenes chilenos disponen de \$134.315 mensuales. Sin embargo, dicho valor varía según género, nivel socioeconómico, tramos de edad y localización urbana-rural. Según el lugar de residencia, las y los jóvenes de ciudades perciben un ingreso medio $47 \%$ superior al de quienes habitan en sectores rurales (Injuv, 2007)

El estudio cualitativo sobre experiencias de las y los jóvenes rurales en el mundo del trabajo (Peña y Gajardo, 2009) evidencia que la relación entre jóvenes rurales de nivel socio-económico bajo y el trabajo está marcada por la precariedad. Respecto de las expectativas sobre el trabajo, en el discurso de las y los jóvenes rurales se evidencia con mayor énfasis que en el caso de los jóvenes urbanos, la centralidad de la satisfacción de necesidades básicas relacionadas con la sobrevivencia y el deseo de tener estabilidad laboral, entendida como blindaje frente a la situación de pobreza y garantía para el futuro; se expresa también la constatación de la existencia de salarios insuficientes para realizar un proyecto de vida autónomo:

"Yo espero siempre el poder tener la plata suficiente a fin de mes para poder pagar todas las cosas que debemos..." (H2, rural).

"Y es bueno saber, que tu trabajo es algo que no se va a acabar así como así... que tiene que pasar algo bien grande para que se acabe, tener esa seguridad te sirve, porque te tranquiliza, te permite hacer un poco de planes a futuro también..." (H2, rural). 
"Es que acá el trabajo no es seguro, porque en cualquier momento reducción de personal y vamos cortando cabezas" (M1, rural).

"A mí lo que siempre me da miedo es no tener en qué trabajar, porque las pegas no son seguras y nada te asegura que vai a seguir trabajando en eso que estái haciendo, por muy bien que lo hagas, si no tienen para pagarte, perdiste no más..." (M5, rural).

"Obviamente uno espera que te paguen bien, que te den una paga acorde a lo que haces y si estás todo un día trabajando bajo el sol, muerta de cansancio, querí que te paguen bien po"' (M4, rural).

"Yo solo espero el sueldo a fin de mes... en serio no espero más..." (M5, rural).

"Es que si uno no trabaja no tiene para darle a sus hijos, no tienes para comprarle aunque sea un engañito para la Navidad y esas cosas duelen, porque veí a tu cabro chico triste por no recibir algo..." (H3, rural).

Otros aspectos problemáticos de la inserción laboral de las y los jóvenes rurales, tienen que ver con la distancia geográfica que hay que recorrer para acceder a las ofertas de trabajo:

“Lo que más cansa en realidad es tener que viajar tanto rato para encontrar un trabajo que te guste... y la distancia no tiene solo que ver con tomar un bus y demorarse harto... también es plata que uno gasta... por ejemplo, una vez trabajé en un trabajo en Santiago centro, pero se me iba todo el sueldo en pasajes... no podía ni mantener a mi familia..." (H3, rural).

"Vivir lejos es un problema, si es que quieres trabajar en algo que no sea lo típico que tienen para ofrecer acá, vivir acá es un problema..." (M4, rural).

Hay otros aspectos de la oferta de trabajo en el sector rural que reflejan en alguna medida un choque con las expectativas de las y los jóvenes y con el nivel educativo alcanzado:

“A mí no me gusta trabajar en las típicas cosas que se pueden trabajar acá, quizás donde veo la realidad de afuera, de la ciudad o de mis propios compañeros... a mí no me gusta que se trate mal a la gente o que se abuse de sus necesidades de tener plata, yo sé que estas no son pegas que mentalmente te pidan mucho, pero lo físico también es importante y abusar de las personas no me parece para nada..." (M4, rural) 


\section{Ejercicio de los derechos laborales}

Otro indicador para observar la discriminación hacia la juventud en el trabajo es el abuso laboral. El abuso de poder en el trabajo se define como "toda acción o práctica que - a partir de un ejercicio ilegítimo individual o institucional del poder- se traduce en un menoscabo de los derechos y/o la dignidad de los/as trabajadores/as tanto al interior de una institución pública o privada, como en las relaciones laborales que puedan ocurrir fuera de estas instancias" (Genera 2009).

Najarí y Piña (2009) observan que las denuncias realizadas en la Dirección del Trabajo, registradas en el periodo del 2005 al 2007, se concentran prioritariamente en el sector comercio (15\%), así como en actividades inmobiliarias, empresariales y de alquiler (12,3\%); sectores en los cuales se insertan las y los jóvenes no calificados, como se señaló anteriormente.

De otro lado, la encuesta jóvenes y trabajo (Genera, 2009) muestra las opiniones de la población en relación con la naturalización de prácticas en el trabajo donde los aspectos más relevantes tienen relación con la formalización de contratos, el pago de horas extras y los beneficios para los jóvenes que hacen sus prácticas laborales. Al observar los datos se evidencia que la población considera que con mayor frecuencia ocurren prácticas negativas más que positivas hacia los jóvenes.

En los aspectos negativos, el 62\% de las personas encuestadas considera que con alta frecuencia, a los jóvenes se les solicita que modifiquen su presentación personal; el 53\% piensa que las empresas prefieren no contratar mujeres en edad fértil y un $41 \%$ piensa que a los jóvenes se les solicitan tareas que no se les solicitarían a adultos en el trabajo. Solo el 10\% cree que con frecuencia las prácticas laborales son pagadas; solo el $16 \%$ considera que las horas extras se les pagan a los jóvenes; y aproximadamente el $20 \%$ considera que con frecuencia se contrata a jóvenes con apariencias no tradicionales o alternativos y que los jóvenes tienen contrato laboral.

En lo que refiere a la presentación personal de los jóvenes en el trabajo un $60 \%$ de las personas creen que es siempre necesario exigir una determinada presentación personal más formal y aproximadamente un $10 \%$ no cree que sea necesario exigir dichas cuestiones a los jóvenes en el trabajo. 
De otro lado, el $79 \%$ de los consultados considera que no se respetan los derechos laborales de los jóvenes, el 61\% considera que se respetan más los derechos laborales de los adultos que los de los jóvenes y el 30\% cree que no se respetan los derechos laborales de los jóvenes y tampoco los de los trabajadores adultos.

Al revisar el discurso de los jóvenes entrevistados en el estudio de Peña y Gajardo (2009), respecto de los abusos en el trabajo, los temas más recurrentes son los horarios de trabajo, el mal trato y las remuneraciones:

"Entrábamos a las dos de la tarde y salíamos a las tres de la mañana, casi doce horas, y solo te ponían un hervidor para que te tomaras una taza de té, mientras llegaba el camión, porque por ejemplo trabajabas así, y no te dejaban ir al comedor a tomarte un té, porque tenías frío, era como muy posesivo el trabajo, había que terminarlo" (M1, rural).

"Muchas veces pasó que nos decían: "Ya, a fin de mes o en tal fecha les pagamos"... pero eso nunca era así, los pagos terminaban siendo cuando los tipos se dignaban a pagarte..." (M4, urbana).

"La colación se supone que es un horario de descanso, no solamente para comer, es tambien un tiempo de descanso para tu trabajo, entonces la idea es que esté en el medio de tu jornada laboral, entonces pasa eso de que por conveniencia de la empresa te mandan a cualquier hora y eso molesta" (M1, urbana).

"Y eso de los malos tratos de los superiores en parte también, porque en la mayoría de las veces se considera la jerarquía como "perfección", y si uno, con menor posición jerárquica, hace reparos a las determinaciones de superiores, se malinterpreta... entonces lo toman a mal y hay problemas..." (H3, urbano).

"no hay claridad en los pagos y en general son dos los aspectos en los que te pueden cagar cachai: en los pagos y en la cantidad de horas, si en cómo se distribuyen las horas de trabajo. Y ahí se empieza a jugar mucho con los horarios que tienen los jóvenes como se supone que somos más flexible si tenemos menos cosas que hacer, por eso tienen menos cosas que hacer" (H6, urbano). 
Respecto de la disposición de las y los jóvenes de nivel socioeconómico medio y bajo para ejercer sus derechos laborales, las siguientes citas reflejan que existen algunas nociones respecto de los derechos laborales en el discurso juvenil, sin embargo estas nociones son con frecuencia confusas, o sencillamente la necesidad de trabajar es más relevante que la intencionalidad de mejorar las condiciones de trabajo.

"Conozco mis derechos, ahora los jóvenes aprenden mucho la parte laboral y saben sus derechos, y eso se adquiere mas que hace 10 o 20 años..." (H2, urbano).

"Sí, los conozco... no conozco el código del trabajo a cabalidad, pero sí los artículos básicos que están en todos los trabajos... seas arquitecto, abogado, periodista, obrero de construcción o asesora del hogar..." (H3, urbano).

"La edad siempre es un elemento de discriminación... te ven joven y te sienten inexperto... vulnerable quizás..." (H5, rural).

"Como te decía mi mami estaba enferma, entonces con eso, lo único que nos importaba con mi hermano era trabajar para darle lo mejor a ella, nunca estuve curiosa con lo de los derechos, creo que no me importa mucho..." (M5, rural).

“El contrato es un derecho, es lo más importante, porque con él puedes regularizar verdaderamente tu vida dentro del trabajo..." (H2, rural).

“Las horas extras pagadas son un derecho también, pero nunca lo cumple... uno tiene que andar negociando siempre y terminan dándote la plata diciendo poco menos que fue un favor... cuando la cosa es al revés..." (H2, rural).

"Si, por ejemplo lo regular es que de las ocho de la mañana, a las doce del día, una de la tarde, a las dos de la tarde, y después hasta las cinco y media de la tarde o seis, ese es el horario..." (H1, rural).

"Yo sé que uno tiene que trabajar una cierta cantidad de horas y luego tiene derecho a descansar, pero no estoy muy claro en cuántas son las horas o los tiempo de cada cosa..." (H3, rural). 
"Mis derechos laborales, la verdad es que conozco algunas cosas, no conozco mucho mis derechos laborales y tampoco creo que el promedio de personas chilenas, sepa sobre sus derechos como trabajador..." (M1, urbana).

'Si po', tienen que darte la carta de aviso que es un mes anterior, tienes treinta días para que te echen y pedir pega en otro lado..." (H1, rural).

"Lo que sé es que no te pueden pasar a llevar, por ejemplo insultarte, no te pueden amenazar con que te van a echar sin la carta de aviso, y eso..." (H1, rural).

"Como estoy recién trabajando por así decirlo "en serio"... no conozco bien, ni como debiera mis derechos laborales..." (H4, urbano).

Las siguientes citas, reflejan el reconocimiento de la utilidad y la importancia que representan las instancias de educación en torno a los derechos laborales, para las y los jóvenes entrevistados:

"Sobre todo que te enseñen los derechos porque nunca los sabemos, porque es una deficiencia que tenemos porque al momento de reclamar no tenemos las palabras o el peso de por qué estamos reclamando o qué estamos reclamando, eso es bastante importante y es deficiente en ese momento..." (H1, urbano).

"Cada trabajador contratado, antes de comenzar su primer día de trabajo, recibe el reglamento interno de la empresa y las nociones básicas de lo que hace la empresa y qué hacer en caso de emergencia, que las hemos tenido. Hay capacitaciones en materia de seguridad laboral y medioambiental regularmente, y eso a la larga es beneficioso también para nosotros mismos, ya que genera confianza y tranquilidad en relación a lo que la empresa hace y sucede..." (H3, urbano).

"Me tocó verlo donde estudiaba porque ahí de repente llegaba gente y te comentaba que los estaban estafando, incluso gente mayor y jóvenes también..." (H2, urbano).

"yo cuando quiero saber sobre los derechos, no me queda de otra que revisar el contrato de trabajo o simplemente preguntarle a hueones que estudian derecho o tienen experiencia en el tema..." (H1, urbano). 
Por último, las siguientes citas relevan el conocimiento y confianza de algunos jóvenes frente a las instancias de fiscalización laboral del Estado:

"Nosotros mismos fuimos, sacamos información mediante la inspección del trabajo, íbamos, consultábamos cómo se podía sacar el valor de las vacaciones, el valor para poder sacar nuestro finiquito, todo y poder legalizar y fue así, tuvimos que mediante la Inspección del trabajo que se pagara la cosa..." (M2, urbana).

“O por último vai a la inspección del trabajo, eso siempre funciona, a todo el mundo le funciona... aparte las empresas como que les da miedo cachái que los acusen, si son igual que cabros chicos en ese sentido..." (M3, urbana).

\section{Conclusiones}

Los datos presentados muestran con contundencia la prevalencia en nuestra sociedad, de una mirada discriminatoria hacia las y los jóvenes pertenecientes a los niveles socioeconómicos medios y bajos. Al parecer, los prejuicios frente a este grupo etario hacen parte de nuestros consensos sociales y traspasan los ámbitos educativo y laboral. Esta afirmación resulta muy significativa si tenemos en cuenta que en los debates públicos sobre el trabajo, las alternativas para el mejoramiento de la calidad del mismo, se subordinan fundamentalmente a variables macroeconómicas.

En este sentido, desde el punto de vista de la actoría social, es innegable la necesidad de hacerse cargo del escándalo ético que representan afirmaciones ampliamente aceptadas, tales como, "las mujeres deben dejar el trabajo siempre que estén embarazadas y los jóvenes son flojos o representan una amenaza para los trabajadores adultos".

Un último aspecto concluyente de este artículo, es la necesidad de intencionar la enseñanza de los derechos laborales tanto en la educación media como en la Universidad, dado que el desconocimiento y/o la ambigüedad existente en este aspecto, generan mayor vulnerabilidad frente a un mercado del trabajo restrictivo y poco orientado a propiciar aprendizajes en los más jóvenes. 


\section{Referencias bibliográficas}

Centro de Relaciones Laborales, Trabajo y Diálogo social (2007). Desarrollo del Mercado del Trabajo en Chile: empleo, productividad, relaciones laboral y diálogo social. Santiago, Chile: Fundación estudios laborales.

CEPAL y OIj (2004). La Juventud en Iberoamérica: Tendencias y Urgencias. Santiago, Chile: AUTOR.

CEPAL (2009). Economía del Cambio Climático en Chile. Santiago, Chile: ONU.

CHILE. DIRECCIÓN DEL TRABAJO (2008). Informe de resultados sexta Encuesta Laboral. Santiago, Chile: AUTOR.

CHILE. Instituto nacional de la Juventud. (2006). Quinta Encuesta Nacional de Juventud. Santiago, Chile, INJUV.

CHILE. MINISTERIO DEL TRABAJO Y PREVISIÓN SOCIAL. SERVICIO NACIONAL DE CAPACITACIÓN Y EMPLEO (2004). Modelo de Estatutos de Organismo Técnico Intermedio para Capacitación. Recuperado el 3 de Mayo 2009, de http://empresas.sence.cl/site2/OTIC_ARCHIvos/otirestatutos.doc

ESCÁRATE MÜLLER, J., (2004). Mercado laboral en Chile. Buenos Aires: MTEYSS/CEIL.

ESPINOZA, M. (2009). Trabajo decente y protección social. Organización Internacional del Trabajo. Recuperado el 10 de Abril de 2009, de http://www.oitchile.cl/pdf/ publicaciones/ser/ser007.pdf.

GUERRA, MA. I. (2005). Los jóvenes del siglo XXI ¿Para que trabajan? Los sentidos del trabajo en la vida de los jóvenes de sectores urbano populares de la ciudad de México. Revista Mexicana de Investigación Educativa, 10(25), pp: 419-449.

OFICINA INTERNACIONAL DEL TRABAJO (2003). Actividades normativas de la OIT en el ámbito de la seguridad y la salud en el trabajo: estudio detallado para la discusión con miras a la elaboración de un plan de acción sobre dichas actividades. Recuperado el 10 de Abril de 2009, de http:// www.ilo.org/public/spanish/standards/relm/ilc/ ilc91/pdf/rep-vi.pdf.

(2005a). El empleo de los jóvenes: vías para acceder a un trabajo decente. Recuperado el 10 de Abril de 2009, de http://www.ilo.org/public/english/employment/ yett/download/ilcrepes.pdf.

(2005b). Marco de promoción en el ámbito de la seguridad y la salud en el trabajo. Organización Internacional del 
Trabajo. Recuperado el 10 de Abril de 2009, de http://www.ilo.org/public/spanish/standards/ relm/ilc/ilc93/pdf/rep-iv-1.pdf._

(2005c). Trabajo Decente y Juventud, Chile. Recuperado el 10 de Abril de 2009, de http://www.oitchile.cl/pdf/ informe\%20chile.pdf.

(2007). Trabajo Decente y Equidad de Género para América Latina, Desafíos para la Igualdad en el Trabajo: Chile. Recuperado el 10 de Abril de 2009, de http://www.oitchile.cl/genero/Chile.pdf.

Weller, J. (2006). Inserción laboral de jóvenes: expectativas, demanda laboral y trayectorias. Recuperado el 15

de marzo de http://www.redetis.org.ar/media/ document/weller.pdf

(2006). Jóvenes, Pobreza y Dinámica Demográfica: El Eslabón del Mercado del Trabajo. Recuperado el 10 de Abril 2009 , de http://www.eclac.org/celade/agenda/5/26955/ PobyPobreza_agenda.pdf.

(2007). La inserción laboral de los jóvenes: características, tensiones y desafíos. Revista de la CEPAL, (92), pp. 61-82. 